\title{
CONTROLLED SURGERY WITH TRIVIAL LOCAL FUNDAMENTAL GROUPS
}

\author{
Erik Pedersen, Frank Quinn and Andrew Ranicki
}

\begin{abstract}
We provide a proof of the controlled surgery sequence, including stability, in the special case that the local fundamental groups are trivial. Stability is a key ingredient in the construction of exotic homology manifolds by Bryant, Ferry, Mio and Weinberger, but no proof has been available. The development given here is based on work of M. Yamasaki.
\end{abstract}

In this note we provide a proof of the controlled surgery exact sequence used in the construction of exotic homology manifolds by Bryant, Ferry, Mio and Weinberger, $[\mathrm{BFMW}]$. A primitive version of controlled surgery was developed by the second author in his definition of the invariant that identifies exotic homology manifolds, [Q3,Q4]. Surgery with bounded control, including exact sequences, was developed in $[\mathrm{FP}]$. The remarkable limit construction of $[\mathrm{BFMW}]$ uses a refinement of the sequences of [FP]. Roughly speaking [FP] describes a limit as $\epsilon \rightarrow 0$ while [BFMW] depends crucially on a stability property of the limiting process. The proof of the refinement was postponed to a planned project that was never completed. The intent was to deduce stability in general from a special case with an independent solution known to be stable, the " $\alpha$ approximation theorem" of $[\mathrm{CF}]$. This is reasonable in principle and may be possible, but it has become clear that the authors of [BFMW] have not addressed serious technical issues needed to actually carry it out. As noted in the review [R3] Until [the planned project] or some appropriate substitute becomes available the surgery classification must be regarded as somewhat provisional - although there is little doubt among the experts that it is correct. The purpose of this paper is to provide the "appropriate substitute". Our proof is direct, and is based on work of Yamasaki [Y].

The following is Theorem 2.4 of [BFMW] with minor inaccuracies corrected.

1. Theorem. Suppose $B$ is a finite dimensional compact metric ANR and a dimension $n \geq 4$ is given. There is a stability threshold $\epsilon_{0}>0$ so that for any $\epsilon_{0}>\epsilon>0$ there is $\delta>0$ with the following property: If $f: N \rightarrow B$ is $(\delta, 1)$ connected and $N$ is a compact $n$-manifold then there is a controlled surgery exact sequence

$$
H_{n+1}(B, \mathbf{L}) \rightarrow \mathcal{S}_{\epsilon, \delta}(N, f) \rightarrow[N, \partial N ; G / T O P, *] \rightarrow H_{n}(B ; \mathbf{L})
$$

1991 Mathematics Subject Classification. 57R67, 57P99.

Key words and phrases. Controlled surgery, homology manifolds.

Partially supported by the US National Science Foundation, EPSRC Grant GR/M82158/01 and the Leverhulme Trust. 


\section{Definitions.}

(1) $\mathcal{S}_{\epsilon, \delta}(N, f)$ is the controlled structure set and is the object of interest. It is defined as the set of equivalence classes of $(M, g)$, where $M$ is a topological manifold and $g: M \rightarrow N$ is a homeomorphism on boundaries and a $\delta$ homotopy equivalence rel boundary. The equivalence relation is given by: $(M, g) \sim\left(M^{\prime}, g^{\prime}\right)$ if there is a homeomorphism $h: M \rightarrow M^{\prime}$ whose restriction to the boundary commutes with $g$ and $g^{\prime}$, and which $\epsilon$ homotopy commutes on all of $M$. It is part of the assertion that this does actually give an equivalence relation.

(2) Sizes refer to measurements in $B$. For instance an $\epsilon$ homotopy between maps $M \rightarrow N$ is $G: M \times I \rightarrow N$ so that for any $x \in M$, the arc $f G(\{x\} \times I)$ lies in the ball of radius $\epsilon$ about $f G(x, 0)$. For basic definitions see Quinn [Q1].

(3) $[N, \partial N ; G / T O P]$ is the set of homotopy classes of maps of $N$, rel boundary, to the classifying space $G / T O P$. This is the same term as appears in the uncontrolled surgery sequence.

(4) $H_{*}(B ; \mathbf{L})$ is homology with coefficients in the 4-periodic simply-connected surgery spectrum $\mathbf{L}$, with $\pi_{*}(\mathbf{L})=L_{*}(\mathbf{Z})$ for $* \geq 0$. The spectrum can be constructed algebraically using (uncontrolled) quadratic Poincaré chain complexes over the ring $\mathbf{Z}$ ([R2]).

\section{Outline.}

The proof divides into two parts. First there is a sequence with more primitive obstruction terms:

$$
L_{n+1}(B ; \mathbf{Z}, \epsilon, \delta) \rightarrow \mathcal{S}_{\epsilon, \delta}(N, f) \rightarrow[N, \partial N ; G / T O P, *] \rightarrow L_{n}(B ; \mathbf{Z}, \epsilon, \delta) .
$$

This is "well known" and quickly assembled from pieces in the literature, though not entirely straightforward. The second part shows that an assembly map defined by Yamasaki,

$$
H_{n}(B ; \mathbf{L}) \rightarrow L_{n}(B ; \mathbf{Z}, \epsilon, \delta)
$$

is an isomorphism for suitable $\epsilon, \delta$. Theorem 1 follows by using this to replace the obstruction term in the primitive sequence. The proof of assembly isomorphism follows that of Yamasaki for the $L^{-\infty}$ case, with the substitution of a $K$-theory splitting argument for stabilization by $\times S^{1}$.

We remark that the key feature needed in constructing homology manifolds is "stability": the sequence in Theorem 1 holds for particular $\epsilon$ and $\delta$ rather than just for the $\epsilon \rightarrow 0$ limit, even though the homotopy and obstruction terms are explicitly independent of $\epsilon$ and $\delta$. Stability in the sequence comes from stability in the assembly isomorphism. Yamasaki's arguments are stable for relatively straightforward reasons, so the basic source traces back through the $K$-theory splitting argument to stability of vanishing of controlled Whitehead groups in [Q1, §8]. Finally we note that for stability the use of $\epsilon$ control, rather than bounded or continuous control, is essential. Bounded versions can be used to identify the inverse limit as $\epsilon \rightarrow 0$, but do not give information on how the limit is approached.

\section{Surgery.}

We describe how the "primitive" surgery sequence of $\S 3$ is a straightforward controlled version of the standard sequence. The only awkward point is that the 
surgery obstruction $[N, \partial N ; G / T O P] \rightarrow L_{n}(B ; \mathbf{Z}, \epsilon, \delta)$ still must be defined using surgery rather than by a direct chain-level construction.

$L_{n}(B ; \mathbf{Z}, \epsilon, \delta)$ is defined to be the group of $n$-dimensional quadratic Poincaré complexes ( $\mathbf{Z}$ coefficients, [R1]), over $B$ and with radius $<\delta$, modulo bordism through $(n+1)$-dimensional quadratic Poincaré pairs with radius $<\epsilon$. Here we use the routine version of controlled algebra [Q1] that locates bases for modules at points in $B$, and measures radii of ordinary homomorphisms in terms of distance between involved basis points. Yamasaki goes further in using "geometric" morphisms that incorporate paths in a space. This refinement is unnecessary here because the local fundamental groups are constant (in fact trivial).

Suppose $M \rightarrow N$ is a normal map representing an element in [ $N, \partial N ; G / T O P]$. For any $\delta>0$ we can do surgery below the middle dimension [Q3] to make $M \rightarrow N$ $(\delta, j)$-connected over $B$, where $n=2 j$ or $2 j+1$. In this case we can give the relative chains a $\delta$ controlled quadratic Poincaré structure: nondegenerate forms with a symmetry condition in even dimensions, and "short odd complexes" $[\mathrm{R} 4, \S 6]$ in odd dimensions. We define the surgery obstruction function by taking a normal map to the quadratic Poincaré structure on a highly-connected normal map in the bordism class.

The verification that this function is well-defined uses a relative construction. If there is a normal bordism between highly-connected normal maps then do surgery to make the bordism also $\delta$-connected below the middle dimension. The relative chains of the bordism can then be given the structure of a $\delta$ quadratic Poincaré chain bordism between the chains of the two maps.

The basis for exactness of the sequence is: if the quadratic structure on the chains of a highly connected normal map is null-bordant through a highly-connected quadratic chain bordism, then we get the usual algebraic data for doing middledimensional surgery to get an equivalence. More specifically if $\epsilon>0$ then there is $\delta>0$ so that if the quadratic chains of a highly $\delta$-connected normal map is highly $\delta$-connected algebraically nullbordant, then the normal map is bordant (by surgery) to an $\epsilon$ equivalence. The $\epsilon$ and $\delta$ here come from the controlled Hurewicz and Whitehead theorems [Q1, §5] rather than subtle stability issues.

This is the point at which dimension issues arise. Standard surgery requires dimension $\geq 5$, and gives surgery sequences for smooth and $P L$ manifolds when the structure set and homotopy terms are changed appropriately. The topological version holds in dimension 4: since the local fundamental groups are trivial [Q2] gives the controlled embeddings of 2-spheres in 4-manifolds needed for the surgery. In fact there is a weak 3-dimensional version in which objects in the structure set are $\delta$ homology equivalences and equivalences are $\epsilon$ homology $s$-cobordisms, but we have not tried to include this in the main statement.

The algebra and topology are brought together by algebraic surgery on controlled quadratic complexes. The results needed are exactly analogous to the topological case, and easily obtained by adding control to the arguments of [R1]: given $n$ and $\epsilon>0$ there is $\delta$ so that an $n$-dimensional $\delta$ quadratic Poincaré complex over $B$ is $\epsilon$ bordant to one that is connected up to the middle dimension. Similarly if two highly-connected quadratic complexes are $\delta$-bordant then there is a highly connected $\epsilon$-bordism.

\section{Assembly.}

The assembly map $H_{n}(B ; \mathbf{L}) \rightarrow L_{n}(B ; \mathbf{Z}, \epsilon, \delta)$ is defined by Yamasaki [Y, §3] 
using "cycles". The characterization of assembly maps in Weiss and Williams [WW] shows this agrees with other definitions. We review $[\mathrm{Y}]$ to explain how it reduces our case to a $K$-theory splitting problem.

An element of $H_{n}(B ; \mathbf{L})$ is represented by (1) a triangulated codimension 0 submanifold $V$ of some sphere $S^{N} ;(2)$ a quadratic Poincaré pair over each simplex of $V$ so that the pairs over $\partial \sigma$ fit together to give the boundary of the pair over $\sigma$, and so that they all glue together to give a Poincaré complex of dimension $n$; and (3) a map $V \rightarrow B$. The complex obtained by glueing them all together is the assembly. To get control on the assembled complex one subdivides $V$ so finely that images of simplices have diameter $<\delta$ in $B$. Define a function from the basis of the assembled complex into $B$ as follows: each basis element in the assembled complex comes from one of the fragments, lying over some simplex. Take the basis element to an arbitrary point in the image of the simplex. Since the structure maps in the assembled complex keep this basis element inside its fragment, therefore over points in a small image, the assembled complex has radius $<\delta$.

This description of the assembly makes it clear that to show it is an isomorphism we need to start with a controlled complex and split it up as a union of small pieces. Full splitting follows inductively from being able to split once, so we are reduced to the following analog of [Y, Lemma 2.5]:

6. Lemma: splitting Poincaré complexes. Suppose $B$ is a metric space, $W \subset$ $B, \epsilon>0$, and a dimension $n$ are given. Then there is $\delta>0$ so that an $n$ dimensional quadratic Poincaré complex $D$ over $B$ with radius $<\delta$ is $\epsilon$ equivalent to a union of $\epsilon$ Poincaré pairs $D^{\prime} \cup_{C} D^{\prime \prime}$ with $D^{\prime}$ located over $B-W, D^{\prime \prime}$ located over $W^{\epsilon}$, and the common boundary $C$ located over $W^{\epsilon} \cap(B-W)$.

Yamasaki comes close to producing such a decomposition. He produces pairs $C \rightarrow D^{\prime}, C \rightarrow D^{\prime \prime}$ with $D^{\prime}, D^{\prime \prime}$ over $B-W, W^{\epsilon}$ respectively, as desired, but $C$ may be nontrivial over all of $B$. In a nutshell, $D^{\prime \prime}$ is the maximal based subcomplex of $D$ lying over $B-W, D^{\prime}$ is the quotient $D / D^{\prime \prime}$ with basis the complement of the basis of $D^{\prime \prime}$ in the basis of $D$. Since these are $n$-dimensional complexes of radius $<\delta, D^{\prime}$ lies over $W^{n \delta}$. Finally $C$ is Ranicki's "algebraic boundary" defined as the mapping cone of a duality homomorphism. This homomorphism uses all of $D$ so has no restrictions on its location. However the duality homomorphism is a $\delta$ chain equivalence outside $W^{n \delta} \cap\left(W^{-n \delta}\right)$, so the mapping cone $C$ is $\delta$ contractible there. Over the region where it is contractible Yamasaki uses the usual folding argument to get $C$ concentrated in two adjacent degrees, in which case the boundary homomorphism is an $n \delta$ isomorphism.

Recall that we would be finished if we could show that given $\epsilon$ there is $\delta>$ 0 so that $C$ is $\epsilon$ equivalent to a complex located over $W^{\epsilon} \cap\left(B-W^{-\epsilon}\right)$. The problem is therefore reduced to a problem about $\delta$ isomorphisms. Namely we have a homomorphism $d: C_{j} \rightarrow C_{j-1}$ that is a $\delta$ isomorphism off $W^{\delta} \cap\left(W^{-\delta}\right)$ and we want to split off and discard a contractible summand containing the part outside $W^{\epsilon} \cap\left(B-W^{-\epsilon}\right)$.

It is at this point that our argument diverges from Yamasaki's. Splitting an isomorphism is a controlled $K$-theory problem and generally not possible. Yamasaki stabilizes by multiplying by $S^{1}$. This canonically kills $K$-theory so splitting becomes possible. However it also changes the surgery problem, so he defines $L^{-\infty}$ by factoring out these changes and concludes that the assembly is an isomorphism in this context. In our special case ( $\mathbf{Z}$ coefficients) the controlled $K$-theory vanishes 
and splitting is possible without stabilization.

7. Lemma: splitting isomorphisms. Suppose $B \supset V$ and $\epsilon>0$ are given. Then there is $\delta>0$ so that if $d: A \rightarrow A^{\prime}$ is a $\delta$ homomorphism of $\mathbf{Z}$-modules over $B$ and is a $\delta$ isomorphism over $B-V$ then there are $\epsilon$ automorphisms $H, H^{\prime}$ of $A$, $A^{\prime}$ respectively so that (1) $H, H^{\prime}$ are the identity over $V^{\epsilon}$, and (2) $H^{\prime} d H$ is induced by a bijection of bases over $B-V^{2 \epsilon}$.

This follows easily from [Q1, Theorem 8.4].

We apply this to the homomorphism $d: C_{j} \rightarrow C_{j-1}$ in the boundary complex considered above. Conclusion (1) enables us to extend $H, H^{\prime}$ by the identity on the rest of $C$ to get an $\epsilon$ equivalence of quadratic Poincaré complexes. Conclusion (2) shows that the new complex is the sum of a trivial complex $(d=1)$ and one lying over $B-V^{2 \epsilon}$. Deleting the trivial summand gives a splitting satisfying the conclusions of Lemma 6. This proves Lemma 6, which shows that the assembly $H_{n}(B ; \mathbf{L}) \rightarrow L_{n}(B ; \mathbf{Z}, \epsilon, \delta)$ is an isomorphism and so completes the proof of Theorem 1.

\section{REFERENCES}

[BFMW] J. Bryant, S. Ferry, W. Mio and S. Weinberger, Topology of homology manifolds, Annals of Maths. 143 (1996), 435-467.

[CF] T.A.Chapman and S.Ferry, Approximating homotopyequivalences by homeomorphisms, Amer. J. Math. 101 (1979), 583-607.

[FP] S.Ferry and E.K.Pedersen, Epsilon surgery theory, Novikov conjectures, index theorems and rigidity, Vol. 1, LMS Lecture Notes, vol. 226, Cambridge, 1995, pp. 167-226.

[Q1] F.Quinn, Ends of maps I., Annals of Maths. 110 (1979), 275-331.

[Q2] _ Ends of maps III: dimensions 4 and 5, J. Diff. Geo. 17 (1982), 503-521.

[Q3] Resolutions of homology manifolds, and the topological characterization of manifolds, Invent. Math. 72 (1983), 267-284.

[Q4] An obstruction to the resolution of homology manifolds, Michigan Math. J. 34 (1987), 284-291.

[R1] A.Ranicki, The algebraic theory of surgery, Proc. Lond. Math. Soc. 40 (1980), 87-283.

[R2] Algebraic L-theory and topological manifolds, Tracts in Mathematics, vol. 102, Cambridge, 1992.

[R3] - Featured Review of [BFMW], Mathematical Reviews 97 (1997), MR97b:57017.

[R4] An introduction to algebraic surgery, Surveys on Surgery Theory: Volume 2, Annals of Mathematics Study, vol. 149, Princeton, 2001, pp. 81-163.

[WW] M.Weiss and B.Williams, Assembly, Novikov conjectures, index theorems and rigidity, Vol. 2, LMS Lecture Notes, vol. 227, Cambridge, 1995, pp. 332-352.

[Y] M.Yamasaki, L-groups of crystallographic groups, Invent. Math. 88 (1987), 571-602.

Dept. of Mathematical Sciences

Binghamton University

Binghamton, New York 13901, USA

E-mail address: erik@math.binghamton.edu

Dept. of Mathematics

Virginia TECH

Blacksburg VA 24061-0123, USA

E-mail address: quinn@math.vt.edu

Dept. of Mathematics and Statistics

UNIVERSITY OF EDINBURGH

Edinburgh EH9 3JZ, Scotland, UK

E-mail address: aar@maths.ed.ac.uk 